\title{
Perception of acoustic iterance: Pitch and infrapitch
}

\author{
RICHARD M. WARREN and JAMES A. BASHFORD, JR. \\ University of Wisconsin, Milwaukee, Wisconsin 53201
}

\begin{abstract}
Detection of acoustic repetition is considered as a continuum extending from .5 through $16,000 \mathrm{~Hz}$. Perceptual characteristics are mapped for the entire range, using repeated randomly derived waveforms (segments from Gaussian noise) as model stimuli. Contributions from temporal domain (neural periodicity) analysis extend from about .5 through $5,000 \mathrm{~Hz}$ and from frequency domain (neural place) analysis from roughly 50 through $16,000 \mathrm{~Hz}$. Within the range of overlapping analyses (50 through $5,000 \mathrm{~Hz}$ ), it is difficult to separate the effects of temporal cues from place cues. However, by using low-frequency acoustic iteration from 1 through $16 \mathrm{~Hz}$, we were able to study temporal analysis in the absence of place cues to repetition. New perceptual phenomena are reported for the "infrapitch" produced by "infratones," some of which are analogous to phenomena observed for the pitch produced by tones. It appears useful for theory to consider pitch and infrapitch as a single topic: the perception of acoustic iterance.
\end{abstract}

Waveforms excised from Gaussian noise and repeated without pauses are useful as model periodic stimuli. Such repeated noise segments (which will be called recycled Gaussian noise or RGN) have no a priori restrictions concerning power and phase spectra, and thus represent a general case for determining general rules governing perception of acoustic repetition or iterance.

As we shall see, experiments with RGN have suggested that perception of acoustic iterance represents a continuum extending well below the tonal limit, ranging from about .5 through $16,000 \mathrm{~Hz}$ (repetition periods from about $2 \mathrm{sec}$ through $.06 \mathrm{msec}$ ). Two types of neural analyses appear to operate within this range: temporal domain or periodicity analysis from roughly .5 through $5,000 \mathrm{~Hz}$, and frequency domain or place analysis from roughly 50 through $16,000 \mathrm{~Hz}$.

Low-frequency RGNs from about .5 through $4 \mathrm{~Hz}$ sound like periodic "whooshing," and from about 4 through $20 \mathrm{~Hz}$ sound like "motorboating" (Guttman \& Julesz, 1963). The noisy or hiss-like quality associated with lower frequencies disappears for RGNs at about $100 \mathrm{~Hz}$ (Warren \& Bashford, 1978). At higher frequencies, in keeping with other complex tones, pitch is determined by the period, and timbre is determined by the particular harmonic structure of individual RGNs.

Let us look more closely at the RGNs below the tonal range, or "infratonal" RGNs, ${ }^{1}$ first studied by Guttman and Julesz (1963). An RGN of, say, $1 \mathrm{~Hz}$

This study was supported in part by a grant from the National Science Foundation (BNS 79-12402) and in part by funds provided by the Graduate School and the College of Letters and Science at the University of Wisconsin-Milwaukee. Requests for reprints should be directed to Richard M. Warren, Department of Psychology, University of Wisconsin, Milwaukee, Wisconsin 53201. has a line spectrum spanning all audible frequencies, with successive harmonics separated by $1 \mathrm{~Hz}$. The ear's spectral analyzing power is limited (see Plomp, 1964) and cannot resolve such closely spaced components. The interaction of the many unresolved harmonics stimulating the same locus on the basilar membrane produces complex local patterns of amplitude fluctuation that are repeated once each second. The change of amplitude with time differs at separate loci, but each of these different patterns has the same period. Information concerning this low-frequency periodicity seems to be available along the entire length of the basilar membrane, for, when we swept a $1 / 3$-octave filter through a broadband infratonal RGN, listeners could hear an infrapitch periodicity corresponding in frequency to that of the iterated acoustic waveform at all center frequencies of the filter. It should be noted that infratonal acoustic iterance is detected solely through temporal information without the concurrent place information characteristic of the tonal range.

We designed Experiment 2 to determine whether such a "pure" neural temporal analysis in the infratonal range follows rules observed for the mixed temporal and place analysis occurring in the tonal range. But, before undertaking Experiment 2, it was considered desirable to complete the description of RGNs of different frequencies by studying the unexplored range from 20 through $100 \mathrm{~Hz}$. Experiment 1 provides information to fill this gap.

\section{EXPERIMENT 1: \\ Perceptual Categories and Boundaries for Low-Frequency Pitch}

When we explored perception of RGNs between 100 and $.5 \mathrm{~Hz}$ in a preliminary study, we could find 
no distinct threshold value for pitch. The RGN became progressively more hiss-like with decreasing frequency. Somewhere between 100 and $50 \mathrm{~Hz}$, the RGN underwent a transition from a smooth, homogeneous signal to one that sounded rough and seemed to pulsate at the repetition rate. Within this transition range, it sometimes was possible to change at will from an "analytic" mode producing the rough sensation with a temporally fine structure to a "synthetic" mode producing the smooth unitary percept. Further decrease in frequency caused the pulsing sound gradually to lose its tonal quality. While Guttman and Julesz (1963) described RGNs as sounding like "motorboating" from 19 through $4 \mathrm{~Hz}$ and as sounding like "whooshing" from 4 through 1 or $.5 \mathrm{~Hz}$, no formal data were presented for placing this transition at $4 \mathrm{~Hz}$. The listeners in our laboratory also heard the boundary between motorboating and whooshing at about $4 \mathrm{~Hz}$. However, the transition seemed to take place over an octave or more, and any values within this range seemed acceptable to our listeners.

It should be noted that detection of RGN iterance requires no special training. It can be heard clearly within a few seconds by inexperienced listeners for any frequency greater than $1 \mathrm{~Hz}$.

\section{Method}

Subjects. Six subjects were used, each of whom had training in auditory research experimentation, music, or both. They all had heard RGNs of different frequencies before the start of the experiment.

Stimuli. The stimuli were produced by repetition of white-noise segments. Output voltage from a white-noise generator was sampled every $20 \mu \mathrm{sec}$ and coded in 12-bit form by a digital delay line built to our specifications by the Physical Data Company. Maximum storage was 600,000 bits, corresponding to a 1 -sec delay when the delay line was operating at its maximum bandwidth of $16,000 \mathrm{~Hz}$. By using a frequency synthesizer as an external clock, it was possible to control the stepping rate of the delay line's shift registers. Input was bandpassed from 50 through $16,000 \mathrm{~Hz}$. By closing a "recycle switch," input to the delay line was rejected, and the signal was looped or recirculated indefinitely in digital form. Digital-to-analog conversion produced an RGN with a period determined by the number of shift registers placed in the circuit and the external clock rate. An internal antialiasing filter reduced spectral artifacts corresponding to digital-to-analog conversion, and reduced transients corresponding to the closing of the digital loop (such transients could not be heard by listeners or seen on sound spectrographs). A low-pass filter (48 dB/octave cutoff) limited the frequency of signals delivered to the matched TDH-49 headphones to $8,000 \mathrm{~Hz}$, producing a sharp cutoff at the upper limit of the headphones' flat response.

RGN repetition rates from 1.22 through $300 \mathrm{~Hz}$ were used, corresponding to periods from 823 through $3.33 \mathrm{msec}$, respectively. This range was spanned in 13 neighboring subranges, each having limits with a repetition ratio of $1: 1.527$. The lowest subrange, for example, extended from $1.22-1.86 \mathrm{~Hz}$, the next from $1.86-2.84 \mathrm{~Hz}$, etc. The subjects were free to adjust the repetition rate within each subrange by turning a knob that changed the clock frequency controlling the stepping rate of the shift registers. Each time the subrange was changed by the experimenter, a new waveform was captured from the noise generator with the delay line clock-rate set at the lowest frequency of the subrange. Adjustments by subjects within a subrange caused temporal expansion or contraction of the repeated waveform. Stimuli were heard diotically at $75 \mathrm{~dB}$ SPL.
Procedure. The subjects were tested while seated in an audiometric room. There were two sessions, each lasting about $30 \mathrm{~min}$. In the first session, the subjects chose the RGN repetition rate corresponding to their lower limit for pitch (i.e., they judged that a decrease below this value did not produce a change in any pitchlike quality of the $R G N$ ), and also selected the $R G N$ repetition rate within the pitch range corresponding to the transition from a smooth, homogeneous sound to a rough, pulsed sound. Each type of judgment was made twice: once with presentation of subranges by the experimenter in order of increasing repetition rates (initial subrange setting at maximum value) and once with presentation of subranges in the opposite order (with minimum initial settings). The subjects varied the repetition rates within successive subranges until the criterion value was reached by adjustment within a subrange. The experimenter then recorded the clock frequency driving the delay line, which was used to calculate the transition-threshold judgment. Judgments of the pitch/infrapitch boundary were alternated with judgments of the smooth/pulsating boundary, and presentation orders were balanced within the group of subjects. During the second testing session on a subsequent day, each subject made the same judgments, with presentation orders reversed with respect to those received in the first session.

\section{Results and Discussion}

All the subjects found it possible to make judgments of the pitch/infrapitch and smooth/pulsing category boundaries for RGNs. Each of the subjects reported that the qualitative changes corresponding to both types of transitions varied gradually with repetition frequency, with some perceptual ambiguity at the values selected for transitions.

The mean threshold values obtained in Experiment 1 are shown in Table 1. These values, together with earlier information for other $\mathrm{RGN}$ repetition rates (data for the infrapitch.range reported by Guttman \& Julesz, 1963; data for the portion of the pitch range above $100 \mathrm{~Hz}$ reported by Warren \& Bashford, 1978) were used to construct Figure 1, which describes the perceptual characteristics for the entire range of readily detectable $\mathrm{RGN}$ repetition rates. ${ }^{2}$ While boundaries separating the different perceptual qualities are represented diagrammatically at single frequencies, it should be emphasized that all transitions are gradual and occur in the vicinity of the indicated frequencies. Within the transitional range, it is often possible to direct attention to one side of the boundary or the other. Tones above $5,000 \mathrm{~Hz}$ are

Table 1

Repetition Frequencies (in Hertz) Corresponding to Transitions in Perceptual Quality for Repeated Gaussian Noise Segments

\begin{tabular}{ccccc} 
& \multicolumn{4}{c}{ Mean Frequency } \\
\cline { 2 - 4 } Transition & \multicolumn{3}{c}{ Order } & SE \\
\cline { 2 - 5 } & $\mathrm{I}$ & $\mathrm{D}$ & $\mathrm{C}$ & 2.1 \\
Infrapitch/Pifch & 18.7 & 22.5 & 20.5 & 11.7 \\
Pulsing/Smooth & 70.5 & 74.0 & 72.2 & 110 \\
\hline
\end{tabular}

Note-The means for increasing (I) order and decreasing (D) order of frequency presentation each represent 12 judgments ( 2 for each of the six subjects), with a total of 24 judgments for the combined (C) means. 


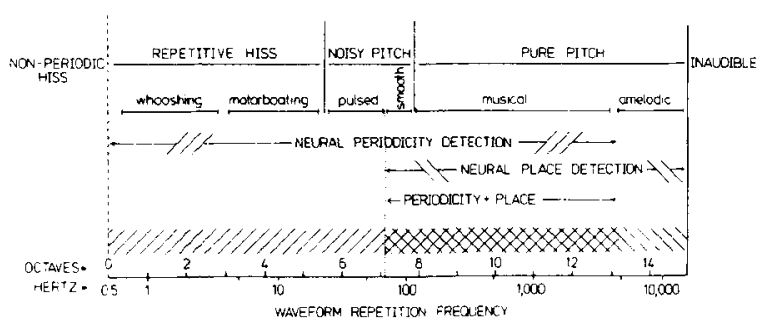

Figure 1. Detection of acoustic iterance for model stimuli consisting of repeated segments of Gaussian noise. Iterance is designated in $\mathrm{Hz}$ and also as the number of octaves above the lower frequency limit for detection. The upper portion of the figure describes the perceptual characteristics: Transitions between the qualities given are gradual, and category boundaries are diffuse, with limits approximately as shown. The lower portion of the figure describes the probable neurological mechanisms operating at particular repetition frequencies, with diagonal lines slanting downward to the left for temporal domain (neural periodicity) and diagonal lines slanting downward to the right for frequency domain (neural place). See the text for further details and discussion.

considered amelodic in Figure 1, since musical intervals cannot be identified for frequencies above this limit (Bachem, 1948; Ward, 1954). Figure 1 also indicates the frequency ranges of neural mechanisms underlying the continuum of acoustic periodicity detection. The literature indicates that the loci of excitation maxima along the basilar membrane provide direct information concerning the frequency of spectral components of the stimulating sound from a diffuse lower limit of roughly 50 through about $16,000 \mathrm{~Hz}$ (Békésy, 1960). The discharge of auditory nerve fibers associated with these loci maintain some degree of synchrony with the local stimulus frequency up to about 4,000 or $5,000 \mathrm{~Hz}$ (Hind, 1972; Rose, Brugge, Anderson, \& Hind, 1967), so that there also can be neural time-domain cues to tonal pitch below that limit. ${ }^{3}$ As was discussed earlier, low-frequency acoustic repetition can be detected only through a neural time-domain analysis. The fundamental frequency of a long period infratonal RGN is below the limit for detectable spectral components, and the harmonic components of the acoustic line spectrum are too closely spaced to permit neural place resolution. Only neural periodicities corresponding to the temporal patterns of amplitude fluctuation along the basilar membrane provide information concerning acoustic iterance.

Hence, the lower infrapitch range provides a rather special opportunity for examining the results of neural periodicity analysis in the absence of neural place involvement. Experiment 2 was designed to examine the results of such an isolated neural periodicity analysis of simultaneous harmonic repetition rates.

\section{EXPERIMENT 2: Perception of Multiple Infratonal Repetition Rates}

When two recycled Gaussian noises are mixed, and each of these RGNs has an identical period but a different randomly derived waveform, the resultant sound is a new recycled noise with the same period. Informal observations had shown that if mixed infratonal RGNs of the same sound-pressure level had repetition rates that differed only very slightly from unison (more than about one part in $10^{3}$ ), then no consistent infrapitch periodicity was heard. ${ }^{4}$ Loss of consistent periodicity also occurred when an infratonal RGN was mixed with on-line noise of equal intensity, indicating that each of the RGNs in the pair mistuned from unison acted as did nonperiodic noise in masking the periodicity of the other. We reasoned that, if the mistuning were increased so that the periodicities had small integral ratios, the harmonic mixture of repetition rates might exhibit one or more detectable periodicities.

RGNs with integral ratios were mixed in Experiment 2 . When the ratio was $1: 2$, the mixture had an ensemble (or fundamental) repetition frequency equal to that of the component with the lower rate. We designed a part of our experiment to determine if this overall repetition rate could be detected and also if the higher harmonic component could be perceived, even though it did not correspond to the waveform repetition frequency. It should be noted that an autocorrelation analysis could reveal the presence of harmonic components, as will be discussed later.

In addition to the RGNs with repetition ratios of 1:2, we also used pairs with ratios of $2: 3$ and $3: 4$. Such mixtures produce a periodic waveform having a frequency differing from both components, with a fundamental or ensemble periodicity corresponding to unity. We wished to determine (1) if either or both harmonic component periodicities could be detected, even though neither corresponded to the frequency of the ensemble waveform, and (2) if the fundamental repetition rate could be detected, even though it was missing from the components. Analogies to each of these types of perception occur in the pitch range, (1) corresponding to the "hearing out" of harmonic components within complex tones (Helmholtz, 1877/ 1954), and (2) corresponding to perception of a pitch equivalent to the fundamental when the fundamental is missing in a complex tone (see Plomp, 1976).

\section{Method}

Subjects. Four subjects were used, each of whom had served in Experiment 1.

Stimuli. The Physical Data digital delay line described in Experiment 1 (maximum signal storage of $1 \mathrm{sec}$ ) was employed together with an Eventide Model 1745A digital delay line (maximum 
total storage of $600 \mathrm{msec}$ with extra storage module) that had a $50-\mathrm{kHz}$ sampling rate and coded the signal in a 10-bit form. The frequency responses of the two delay lines were matched to within $\pm 2 \mathrm{~dB}$ from 50 trrough $12 \mathrm{kHz}$ at the sampling rates employed. In addition to internal antialiasing filters, the output from each delay line was bandpass filtered ( $48 \mathrm{~dB} /$ octave slopes) from 100 to $8,000 \mathrm{~Hz}$. Single infratonal RGNs prepared in this fashion had no audible clicks, and sound spectrograms gave no evidence of transients corresponding to clicks.

A single crystal served as the time base for both delay lines. This clock was located in the Eventide delay line, and its output was used to drive an adjustable Rockland Model 5100 frequency synthesizer, which in turn drove the Physical Data delay line. The frequency synthesizer operated at values close to $600 \mathrm{kHz}$ in driving the Physical Data delay line and could be adjusted in steps of $.001 \mathrm{~Hz}$.

Although each delay line had a frequency response that measured flat to within $\pm 1.5 \mathrm{~dB}$ from 100 through $8 \mathrm{kHz}$ using a Rockland Model $512 S$ spectrum analyzer, in order to minimize spectral bias, RGN captures on each delay line were based on recordings (made previously on the same channel) of white noise produced by the same generator passed through the other delay line set at zero delay. The repetition ratios of members of an RGN pair were accurate, as given in this paper, to at least one part in $10^{8}$. Six sets of component pairs were used: $1+2 \mathrm{~Hz}, 4+8 \mathrm{~Hz}$, $2+3 \mathrm{~Hz}, 3+4 \mathrm{~Hz}, 8+12 \mathrm{~Hz}$, and $12+16 \mathrm{~Hz}$. Experimental stimuli were prepared by recording both RGNs at the same time on separate channels of an Ampex Model $440 \mathrm{C}$ tape recorder: Simultaneous recording prevented random variations in recording speeds from changing the precise integral ratio of repetition rates. ${ }^{5}$ Separate noise captures for each of the six stimulus pairs were used in each of the four experimental sessions. Recordings were used for convenience, but only after preliminary observations indicated that results were similar to those obtained with on-line RGNs."

Listeners matched the RGN periods that they heard, using nonrecycled white noise at $75 \mathrm{~dB}$ SPL, interrupted by 30 -msec silent gaps occurring regularly at a period controlled by a knob that they adjusted. For mixed pairs, component RGNs were each at $75 \mathrm{~dB}$. Mixing was accomplished using a Gately SPM6 mixer.

Procedure. Preliminary experiments indicated that the fundamental period corresponding to the waveform repetition of a harmonic mixture of infratonal RGNs was perceived readily and was the strongest periodicity heard. It was necessary to use considerable caution in choosing an experimental procedure for determining whether or not the harmonic RGNs also could be detected. We could not use the procedure described by Helmholtz (1877/ 1954) for hearing out spectral components of a complex tone by first presenting a spectral component alone and then listening for its continuity when followed immediately by the complex tone at a louder level. When this procedure is used with an RGN (say, $3 \mathrm{~Hz}$ ), followed immediately by a louder mixture of $2-$ and $3-\mathrm{Hz}$ $\mathrm{RGNs}$, the $3-\mathrm{Hz}$ component indeed can be heard readily, along with the dominant $1-\mathrm{Hz}$ periodicity. But the $3-\mathrm{Hz}$ repetition can be heard to continue if followed by a "pure" $1-\mathrm{Hz}$ RGN with no harmonic components, and illusory continuity can be heard even when the 3-Hz RGN is followed immediately by an on-line (nonrepeated) noise.' We also observed that listeners who had had considerable practice listening to harmonic mixtures of RGNs reported perceiving infratonal harmonics within single "pure" RGNs without immediate prior exposure to that harmonic." Experiment 2 was designed so as to minimize the risk of a false demonstration of the ability to detect multiple infratonal repetition rates. Our listeners, while experienced in listening to single RGNs, were kept from prior experience with the multiple infratonal periodicities used in this study. As a further precaution, three separate measures for the detection of harmonic periodicity were used: (1) a report of the number of components heard, (2) the matching of periodicities for each of the components heard, and (3) a report of the duration of continued perception of an RGN, first heard alone, following the addition of a second harmonically related RGN (a brief silence preceded addition of the second RGN)."

The subjects were tested in a sound-attenuating booth and listened to stimuli diotically through matched TDH-49 headphones. They participated in four sessions, each lasting approximately $45 \mathrm{~min}$. During each session, the subjects made three types of judgments with each of the six RGN pairs, finishing all judgments with one pair before moving to the next. The order in which pairs were presented was counterbalanced across subjects and sessions. For their first task with a mixed pair, the subjects listened as long as they wished and then told the experimenter the number of different repetition frequencies that they could detect. For their second task, they matched each of the repetition frequencies heard (in order of increasing frequency) with the interruption rate of an on-line noise. They switched at will between the RGN pair and the noise interrupted by a periodic 30 -msec silent gap, adjusting the interruption rate to match the repetition rate. The experimenter always set the interruption rate at the lower limit of $.1 \mathrm{~Hz}$ before the subject started adjustment. After completing a match, the listener was presented with the component RGNs individually (with orders counterbalanced across sessions) and asked to choose which, if either of them, matched the interruption rate previously selected. (The on-line noise interrupted at the rate just chosen was available to the subject, who could switch between this sound and the single RGN at will.)

The third and final task required listening for several seconds to one component of an RGN pair heard alone and then, when ready, instructing the experimenter to add the other component. All input to the headphones was shut off for $2 \sec$ (see Footnote 9), and the mixture was then presented. The subject kept a timing button depressed whenever the original RGN repetition rate could be heard in the mixture. The criterion for successful detection required the listener to hear the first component in the mixture for 20 continuous seconds, starting within $30 \mathrm{sec}$ after the addition of the other component. ${ }^{10}$ Both members of each RGN pair were judged in this fashion, with the order of presentation counterbalanced across sessions.

\section{Results and Discussion}

When the subjects listened to the stimuli consisting of pairs of RGNs with repetition ratios of $1: 2,2: 3$, and $3: 4$, multiple rates could be heard with each of the three separate tasks. When asked how many repetition rates were heard (the first task), two or more rates were reported for more than $65 \%$ of the stimulus presentations. The second task involved matching of repetition rates, and, as shown in Table 2, matches were generally accurate. The fundamental or ensemble periodicity tended to be dominant and, as can be seen in the table, was matched to within one-half octave or better in almost every trial. Usually, in addition to matching to the fundamental, listeners were able to match to either one or both of the frequencies of the RGN pair. Finally, it was possible to continue to hear an RGN following addition of a second harmonically related RGN (the third task). Continued perception of the target RGN occurred more frequently than is listed in Table 2: Often, this component would disappear and then reappear, and so not meet the criterion for continuous detection necessary for a successful trial. Thus, each of three separate measures used in Experiment 2 indicates that listeners can hear harmonic components in a mixture of infratonal RGNs. 
Table 2

Repetition Frequencies Perceived for Mixed Pairs of Repeated Gaussian Noise Segments

\begin{tabular}{|c|c|c|c|c|}
\hline Pair & $\begin{array}{l}\text { Alone- } \\
\text { Combined* }\end{array}$ & $\begin{array}{c}\text { Acceptable } \\
\text { Matches } \dagger\end{array}$ & $\begin{array}{l}\text { Mean Accept- } \\
\text { able Match }\end{array}$ & SE \\
\hline \multicolumn{5}{|c|}{ Octave Pairs (in Hertz) } \\
\hline $\begin{array}{l}1 \\
2\end{array}$ & $\begin{array}{r}100 \\
56\end{array}$ & $\begin{array}{r}100 \\
50\end{array}$ & $\begin{array}{l}1.02 \\
2.04\end{array}$ & $\begin{array}{l}.014 \\
.148\end{array}$ \\
\hline $\begin{array}{l}4 \\
8\end{array}$ & $\begin{array}{l}94 \\
50\end{array}$ & $\begin{array}{r}100 \\
25\end{array}$ & $\begin{array}{l}3.92 \\
8.89\end{array}$ & $\begin{array}{l}.041 \\
.368\end{array}$ \\
\hline \multicolumn{5}{|c|}{$\begin{array}{l}\text { Harmonic Component Pairs and Their Fundamental } \\
\text { Repetition Frequencies (in Hertz) }\end{array}$} \\
\hline $\begin{array}{l}1 \\
2 \\
3\end{array}$ & $\begin{array}{l}50 \\
25\end{array}$ & $\begin{array}{r}100 \\
63 \\
6\end{array}$ & $\begin{array}{l}1.03 \\
1.95 \\
2.81\end{array}$ & $\begin{array}{l}.017 \\
.034\end{array}$ \\
\hline $\begin{array}{l}1 \\
3 \\
4\end{array}$ & $\begin{array}{l}44 \\
19\end{array}$ & $\begin{array}{l}94 \\
19 \\
38\end{array}$ & $\begin{array}{l}1.03 \\
2.65 \\
4.00\end{array}$ & $\begin{array}{l}.019 \\
.224 \\
.099\end{array}$ \\
\hline $\begin{array}{r}4 \\
8 \\
12\end{array}$ & $\begin{array}{l}63 \\
44\end{array}$ & $\begin{array}{r}100 \\
50 \\
19\end{array}$ & $\begin{array}{r}3.96 \\
9.69 \\
12.00\end{array}$ & $\begin{array}{l}.051 \\
.287 \\
.889\end{array}$ \\
\hline $\begin{array}{r}4 \\
12 \\
16\end{array}$ & $\begin{array}{l}44 \\
31\end{array}$ & $\begin{array}{r}100 \\
50 \\
19\end{array}$ & $\begin{array}{r}4.08 \\
12.70 \\
17.10\end{array}$ & $\begin{array}{l}.064 \\
.243 \\
.480\end{array}$ \\
\hline
\end{tabular}

Note-The fundamental repetition frequencies (in italics) were generated by mixing the pair of harmonic components listed below them. *Percentage of 16 trials 4 trials for each of four subjects) during which the repetition frequency of the component listed, first heard alone, could be detected as continuing for a minimum of $20 \mathrm{sec}$ after being combined with the second component. tPercentage of trials for which matching of the repetition frequency listed was within criterion limits (see text for further details).

\section{ADDITIONAL OBSERVATIONS AND GENERAL DISCUSSION}

Let us consider again the nature of neural place and neural periodicity analyses of acoustic repetition.

Wiener (1930) has shown that an autocorrelational analysis in the time domain can provide the same information provided by a power spectrum analysis in the frequency domain. Both types of analyses appear to occur with tones, but the role of each in various aspects of pitch perception is controversial, partly because either alone can explain many of the phenomena observed (for discussion of theories of pitch perception, see de Boer, 1976; Evans, 1978; Plomp, 1967, 1976).

In the lowest several octaves of detectable acoustic repetition, frequency analysis based upon place cannot provide information concerning the repetition frequency of RGNs. The fundamental and lower spectral harmonics of infratonal RGNs are not audible, and, as discussed earlier, the individual higher spectral harmonics are too closely spaced for resolution by a frequency domain analysis. Only neural patterns in the time domain can provide information concerning acoustic iterance at these low frequencies (see Figure 1).
While a time domain analysis is required for perception of infratonal frequencies, the question can be asked whether such temporal analysis of RGNs is based upon recognition of the recurrence of singularities in the pattern of stimulation or upon a holistic recognition of the repeated pattern. Earlier work with infratonal repetition, using recycled sequences consisting of three or four successive sounds (selected from among sinusoidal tones, square waves, and various noise-derived sounds), indicated that a holistic pattern recognition took place with such sequences when item durations were too brief to permit naming of individual components within the iterated patterns (Warren, 1974; Warren \& Ackroff, 1976; see, also, Warren, 1976). The subjects listening to these repeated sequences could discriminate readily between permuted orders of component sounds. If recurrence of singularities was the only cue to repetition, permuted orders of the same components repeated at the same frequency could not be distinguished, since the repetition of singularities corresponding to the individual components would occur at the same rate with different internal arrangements. Only temporal relations within the patterns were changed with permuted orders. These observations led to a preliminary experiment with RGNs. An RGN with a period of $150 \mathrm{msec}$ was divided into three 50 -msec segments, which we can call $A, B$, and $C$. Using programming equipment and digital delay lines, two RGNs were made available to listeners-one was ABCABCA... and one was ACBACBA.... Even untrained listeners could distinguish between these two patterns, indicating that temporal relations within the patterns could be appreciated readily. It appears that the detection of infrapitch periodicity may be related to what has been called "echoic memory" (Neisser, 1967) or "tape-recorder memory" (Norman, 1972).

In music, "rhythm" can be considered as repetition of special auditory patterns at infratonal frequencies. It is of interest that music plays with acoustic repetition (and systematic variations from strict repetition) for frequencies covering most of the range shown in Figure 1, extending upward through the melodic range to within a few octaves of the limit of audibility, and extending downward in the rhythmic range for a few octaves below the limit for "whooshing" of repeated Gaussian noise segments (the value of $.5 \mathrm{~Hz}$ in Figure 1 is not intended as the lowest detectable iteration frequency for acoustic patterns, even for RGN; see Footnote 2). Music can involve multiple infratonal periodicities of considerable complexity: African polyryhthms use sequences of percussive sounds that can contain several harmonically related rhythmic frequencies, and listeners are able to attend either to the ensemble periodicity or to one or another of the rhythmic components. However, unlike the case with RGNs, rhythmic percussive lines can be followed even when they deviate from harmonic rela- 
tions with other lines. Some African music uses "additive polyrhythms" in which component inharmonic periodicities are each perceived (see Sachs, 1953), while our work has shown that mixing of inharmonic RGNs inhibits detection of any periodicity. ${ }^{11}$

We have seen that consideration of RGNs as model stimuli led to the hypothesis that responses to acoustic periodicities from roughly .5 through $16,000 \mathrm{~Hz}$ represent the single perceptual continuum described by Figure $1 .{ }^{12}$ This hypothesis in turn led to the expectation that some phenomena found in the pitch range may be found for infrapitch as well. Experiment 2 describes the first of these analogs, but we have looked for, and found, others. One of these analogs is infrapitch echo.

When a noise is mixed with its echo having a delay of $\tau \mathrm{sec}$, a pitch corresponding to $1 / \tau \mathrm{Hz}$ can be heard. This "echo pitch" has been reported to occur for delays from about $.02 \mathrm{sec}$ through $5 \times 10^{-4} \mathrm{sec}$, corresponding to pitches from 50 through $2,000 \mathrm{~Hz}$ (for reviews, see Bilsen, 1970; Yost \& Hill, 1978). Theories concerning the basis of echo pitch generally involve the spectral peaks in the power spectra that occur at integral multiples of $1 / \tau \mathrm{Hz}$. Indeed, one of the names for this sound, "rippled noise pitch," refers to its power spectrum.

However, we considered that analysis in the temporal domain might be involved as well, so that infrapitch echo might be detectable. Investigation led to the finding that echo delay of noise can be identified down to at least $2 \mathrm{~Hz}(\tau=.5 \mathrm{sec})$, although pitch is indeed lost at about $50 \mathrm{~Hz}$. Infrapitch echo is marked by an intermittent quality and is similar to (but weaker and more difficult to hear than) RGNs of the same repetition frequency. The spectral peaks of infrapitch echo are too closely spaced along the basilar membrane for a power spectrum analysis based on place of stimulation to be effective, and detection appears to be based on analyses in the temporal domain. Further discussion of infrapitch echo is given elsewhere (Warren, Bashford, \& Wrightson, 1980).

Both Experiment 2 and infrapitch echo involve temporal patterns superimposed on the same cochlear loci. The different patterns of musical polyrhythms also stimulate identical (or overlapping regions) of the basilar membrane. But what would be perceived if harmonically related infrapitch repetitions were delivered to separate loci on the same cochlea with no spatial overlap? In the pitch range, when the lower harmonic components of a complex tone stimulate different loci on the basilar membrane, integration of frequency information takes place, and a single pitch corresponding to the fundamental frequency (or waveform repetition frequency) generally is heard, even when the fundamental is missing from the harmonic sequence of spectral components. The relative contributions of spatial domain and temporal domain information to this integration are a matter of some dispute (for review, see Plomp, 1976). However, we have found that it is possible to examine integration of temporal information at different loci in the absence of place cues to repetition by using harmonic infratonal iterances.

A beating sinusoidal tone pair can be used to produce any frequency of infratonal amplitude modulation at any frequency-sensitive place on the basilar membrane. The difference in frequencies of the pure tones determines the beat rate, and the center frequency of the tone pair determines the place of this infratonal amplitude modulation. Use of several simultaneous pairs of beating tones permits generation of a harmonic sequence of beats (a complex beat), with any desired locus for individual harmonic components. It was found that pooling of the infratonal temporal information took place under all conditions employed, including those involving nonoverlapping widely separated positions on the basilar membrane. Even when the fundamental rate of the complex beat was missing, the ensemble or fundamental infratonal iterance was heard (Warren, Note 1).

Hence, while Experiment 2 and infrapitch echo detection indicate that temporal patterns that are mixed at the same locus can be differentiated, complex beats demonstrate that separate temporal patterns at different loci can be integrated. Temporal domain analysis appears to be quite versatile and capable of producing effects at infratonal frequencies analogous to those encountered with tones.

\section{REFERENCE NOTE}

1. Warren, R. M. Auditory integration of multiple beat rates. Manuscript submitted for publication, 1980 .

\section{REFERENCES}

Bachem, A. Chroma fixation at the ends of the musical frequency scale. Journal of the Acoustical Society of America, 1948, 20, 704-705.

BÉ KÉSy, G. von. Experiments in hearing. New York: McGrawHill, 1960.

BILSEN, F. A. Repetition pitch; its implication for hearing theory and room acoustics. In R. Plomp \& G. F. Smoorenburg (Eds.), Frequency analysis and periodicity detection in hearing. Leiden, The Netherlands: Sijthoff, 1970.

Bilsen, F. A., \& Ritsma, R. J. Repetition pitch and its implication for hearing theory. Acustica, 1969/70, 22, 63-73.

DE BOER, E. On the 'residue' and auditory pitch perception. In W. D. Keidel \& W. D. Neff (Eds.), Handbook of sensory physiology: Auditory system, clinical and special topics (Vol. 5 , Pt. 3). Berlin: Springer-Verlaged 976.

Evans, E. F. Place and time coding of frequency in the peripheral auditory system: Some physiological pros and cons. Audiology, $1978,17,369-420$.

Guttman, N., \& Julesz, B. Lower limits of auditory periodicity analysis. Journal of the Acoustical Society of America, 1963, 35,610 .

Guttman, N., \& Pruzansky, S. Lower limits of pitch and musical pitch. Journal of Speech and Hearing Research, 1962, 5, 207-214.

HELMHOLTZ, H. L. F. VON. On the sensations of tone as a physiological basis for the theory of music (2nd English ed.). New 
York: Dover, 1954. (Translation conformal with 4th German ed., 1877)

Hind, J. E. Physiological correlates of auditory stimulus periodicity. Audiology, 1972, 11, 42-57.

Houtgast, T. Psychophysical evidence for lateral inhibition in hearing. Journal of the Acoustical Society of America, 1972, 51, 1885-1894.

Neisser, U. Cognitive psychology. New York: Appleton-CenturyCrofts, 1967.

Norman, D. A. The role of memory in the understanding of language. In J. F. Kavanagh \& I. G. Mattingly (Eds.), Language by ear and by eye: The relationships between speech and reading. Cambridge, Mass: M.I.T. Press, 1972.

Plomp, $\mathrm{R}$. The ear as a frequency analyzer. Journal of the Acoustical Society of America, 1964, 36, 1628-1636.

Plomp, R. Pitch of complex tones. Journal of the Acoustical Society of America, 1967, 41, 1526-1533.

Plomp, R. Aspects of tone sensation. London: Academic Press, 1976.

Rose, J. E., Brugge, J. F., Anderson, D. J., \& Hind, J. E. Phase-locked response to low-frequency tones in single auditory nerve fibers of the squirrel monkey. Journal of Neurophysiology, 1967, 30, 769-793.

SACHs, C. Rhythm and tempo. New York: Norton, 1953.

WARD, W. D. Subjective musical pitch. Journal of the Acoustical Society of America, 1954, 26, 369-380.

WARREN, R. M. Illusory changes of distinct speech upon repetition - The verbal transformation effect. British Journal of Psychology, 1961, 52, 249-258.

Warren, R. M. Auditory temporal discrimination by trained listeners. Cognitive Psychology, 1974, 6, 237-256.

WarRen, R. M. Auditory illusions and perceptual processes. In N. J. Lass (Ed.), Contemporary issues in experimental phonetics. New York: Academic Press, 1976.

Warren, R. M., \& ACkroff, J. M. Two types of auditory sequence perception. Perception \& Psychophysics, 1976, 20, 387-394.

Warren, R. M., \& Bashford, J. A. Production of white tone from white noise and voiced speech from whisper. Bulletin of the Psychonomic Society, 1978, 11, 327-329.

Warren, R. M., Bashford, J. A., JR., \& Wrightson, J. M. Infrapitch echo. Journal of the Acoustical Society of America, $1980,68,1301-1305$.

Warren, R. M., Obuse k, C. J., \& Ackroff, J. M. Auditory induction: Perceptual synthesis of absent sounds. Science, 1972, 176, 1149-1151.

Wiener, N. Generalized harmonic analysis. Acta Mathematica, $1930,55,117-258$.

YoST, W. A., \& HILL, R. Strength of the pitches associated with ripple noise. Journal of the Acoustical Society of America, $1978,64,485-492$.

\section{NOTES}

1. We will use the terms "tone" and "pitch" in keeping with the following definitions approved by the American National Standards Institute: "A tone is a sound wave capable of exciting an auditory sensation having pitch" and "Pitch is that attribute of auditory sensation in terms of which sounds may be ordered on a scale extending from high to low' (ANSI S3.20-1973). This scale extending from high to low usually is taken as melodic pitch rather than detectable repetition (e.g., Guttman \& Pruzansky, 1962, considered $19 \mathrm{~Hz}$ to be the lowest frequency imparting "pitch" to pulse trains). Therefore, we will consider RGNs with detectable periodicities lying below the limits of musical tonality to be "infratones" producing an "infrapitch" that can be ordered from fast to slow (rather than from high to low).

2. Guttman and Julesz (1963) gave .5 Hz as the lower limit for RGN detection, using as their criterion relatively easy detection achieved with relatively little training. While they gave no specific values, they indicated that a practiced observer could, with effort, achieve detection of repetition at lower frequencies. We found that our best listener could, after considerable training, tap out the period for unknown waveform repetition frequencies down to at least $.1 \mathrm{~Hz}$. These very low frequencies were generated using two recirculating digital delay lines, say, one producing an RGN at $2 \mathrm{~Hz}$ and the other producing an RGN at $2.1 \mathrm{~Hz}$-when the RGNs were mixed, the ensemble waveform was repeated at $.1 \mathrm{~Hz}$.

3. For a detailed discussion of peripheral place and time coding of tonal frequencies and for interesting suggestions concerning their possible interactions, see Evans (1978).

4. It was necessary to use uncorrelated segments of white noise for these observations of mistuned unison. When we recycled the very same noise segment having a repetition rate of $2 \mathrm{~Hz}$ on two separate delay lines and increased the clock frequency driving one of the delay lines to change one of these identical waveforms to $2.2 \mathrm{~Hz}$, then cyclical pitch variations occurred with a period of $.2 \mathrm{~Hz}$ (i.e., once every $5 \mathrm{sec}$ ) when the RGNs were mixed. A rising pitch was heard as the component RGN waveforms moved into alignment, followed immediately by a falling pitch as they moved out of alignment. These glissandos appear to be a form of echo pitch (for discussions of echo pitch or repetition pitch, produced by mixing two sounds of similar or identical waveforms, see Bilsen \& Ritsma, 1969/70; Plomp, 1976). Analogous double glissandos also have been described for mistuned pitch-range RGNs (Warren, Note 1).

5. As with mistuned unisons (ratio 1:1), detection of mixed infrapitch RGNs with periodicity ratios of 1:2, 2:3, 3:4 was quite sensitive to mistuning from integral ratios. Mistuning by more than one part in $10^{3}$ disrupted detection of regular periodicities, and mistuning by one part in $10^{4}$ or $10^{5}$ produced a continual change in the quality of the periodicity heard. Mistuning of one part in $10^{\circ}$ was generally undetectable, and, in the experiment reported, mistuning was well below the limit of detectability.

6. While random variations in the speed of the tape recorder used in Experiment 2 caused perturbations in frequency of about one part in $10^{4}$, such variation did not affect the playback ratio of the frequencies of the RGNs that had been recorded simultaneously, so that their moment-by-moment interaction produced a fixed periodic waveform.

7. This apparent continuity seems to result from a temporal induction, which has been described by Warren, Obusek, and Ackroff (1972) as follows: "If there is contextual evidence that a sound may be present at a given time, and if the peripheral units stimulated by a louder sound include those which would be stimulated by the anticipated fainter sound, then the fainter sound may be heard as present." Since infratonal RGNs and Gaussian noise are broadband, they stimulate all loci along the basilar membrane, and illusory continuity is heard when the RGN is followed by the noise. For a more detailed description of temporal induction and a comparison with the related concept of "pulsation threshold" by Houtgast (1972), see Plomp (1976).

8. It should be noted that illusory continuity is not the only type of temporal induction. Sufficient training or experience can lead to an anticipated sound being heard within any louder sound that could be masking the anticipated signal were it present (see Warren, 1976).

9. The brief silent interval before presentation of the mixture was necessary to inhibit the illusory continuity of a noise through a subsequent temporally contiguous louder noise. Such illusory continuity of a fainter noise has been reported to persist for several tens of seconds (Warren, Obusek, \& Ackroff, 1972).

10. It was sometimes necessary for the listener to "search" for several seconds before teasing out the target periodic component from the mixture-hence, the allowance for initial searching time. Identification of the target was not considered successful unless it could be heard continuously for $20 \mathrm{sec}$, in order to avoid the possibility of a transient misidentification. Preliminary experiments using an initial target RGN, followed (after a .2-sec pause) 
by a harmonically related single RGN ( $3 \mathrm{~dB}$ louder than the target), indicated the effectiveness of the procedure employed for avoiding false identifications of components in mixed RGNs.

11. The differences noted in perception of polyrhythms and mixed infratonal RGNs are not inconsistent with our suggestion that repeated Gaussian noise be considered as a general or model stimulus: That is, the rules governing perception of RGNs apply to other audible periodic sounds at corresponding repetition frequencies; but also, additional rules limited to special repeated sounds may be found. Other complex sounds with infratonal periodicities and special additional characteristics are repeated sequences containing three or four component sounds (Warren, 1974) and repeated single words (Warren, 1961, 1976).
12. It should be noted that specific sounds may not be suitable for periodicity detection over this entire range. Since sinusoidal tones become inaudible below about $20 \mathrm{~Hz}$, they are eliminated as stimuli for low frequencies. Pulse trains at low frequencies are heard as clicks separated by silence, and the only lower limit for detection of repetition is set by the patience of the listener.

(Received for publication November 3, 1980; revision accepted February 23, 1981.) 